\title{
Determination of stellar parameters for Ariel targets: a comparison analysis between different spectroscopic methods
}

\author{
Anna Brucalassi ${ }^{1}$ (D) $\cdot$ Maria Tsantaki ${ }^{1} \cdot$ Laura Magrini $^{1} \cdot$ Sergio Sousa $^{2}$. \\ Camilla Danielski ${ }^{3}$. Katia Biazzo ${ }^{4}$. Giada Casali ${ }^{1,5}$. \\ Mathieu Van der Swaelmen ${ }^{1}$. Monica Rainer ${ }^{1}$. Vardan Adibekyan ${ }^{2}$. \\ Elisa Delgado-Mena ${ }^{2} \cdot$ Nicoletta Sanna $^{1}$
}

Received: 30 June 2020 / Accepted: 15 December 2020 / Published online: 5 March 2021

(C) The Author(s) 2021

\begin{abstract}
Ariel has been selected as the next ESA M4 science mission and it is expected to be launched in 2028. During its 4-year mission, Ariel will observe the atmospheres of a large and diversified population of transiting exoplanets. A key factor for the achievement of the scientific goal of Ariel is the selection strategy for the definition of the input target list. A meaningful choice of the targets requires an accurate knowledge of the planet hosting star properties and this is necessary to be obtained well before the launch. In this work, we present the results of a bench-marking analysis between three different spectroscopic techniques used to determine stellar parameters for a selected number of targets belonging to the Ariel reference sample. We aim to consolidate a method that will be used to homogeneously determine the stellar parameters of the complete Ariel reference sample. Homogeneous, accurate and precise derivation of stellar parameters is crucial for characterising exoplanet-host stars and in turn is a key factor for the accuracy of the planet properties.
\end{abstract}

Keywords Exoplanet atmospheres · Space missions · Optical and IR spectroscopy

\section{Introduction}

So far, more than 4000 planets (with more than 3000 transiting their stars) have been detected showing an incredible diversity in terms of masses, sizes and orbits. Moreover, thousands of Jupiter-size down to Earth-size planets are expected to be

Anna Brucalassi

anna.brucalassi@inaf.it

Extended author information available on the last page of the article. 
discovered in the next few years by Gaia [33, 51], TESS [38], CHEOPS [11] and the upcoming space surveys, such as PLATO [37], along with ground-based surveys, like WASP [35], NGTS [66], TRAPPIST [24], HARPS [29] and ESPRESSO [32], CARMENES [36] and SPIRoU [6]. The recent success of ground-based, space transit and radial velocity searches has ushered exoplanet research into an era of characterisation studies with the goal to investigate the nature, formation, and evolutionary history of the detected objects.

At first, these studies have been focused on understanding the internal structure of exoplanets. From the transit light-curve, the planet radius can be measured and from spectroscopic Doppler measurements, the planet mass is obtained. From the bulk density we have the first hints of the internal structure of the exoplanet and the gas/ice/rock ratios. However, to have a reliable estimate of the density, an accurate knowledge of both radius (with a precision of up to $5 \%$ ) and planetary mass (up to $10 \%$ ) is necessary $[8,65]$. Additionally, the absolute value of planetary radius and mass relies on the precise determination of the radius and mass of the exoplanethost star. The derivation of these last two values is in turn strongly connected to the effective temperature $\left(T_{\text {eff }}\right)$, surface gravity $(\log g)$, and the metallicity of the star. Thus, the planetary properties are critically dependent on their stellar host properties $[2,50,57]$.

Furthermore, transiting planets provide us one of the best ways of characterising their atmospheres. In-transit spectroscopy as well as secondary transit studies [12, 20, 34, 44, 54, 62, 68] using space observatories, as Hubble and Spitzer, and some ground-based observatories, have yielded the detection of some important molecules present in the planetary atmospheres for a limited number of targets, or have identified the presence of clouds, probing the thermal structure and providing some constraints on the planet properties. However, the data available is still too sparse to provide a consistent interpretation and the achieved results point out the main limitations of the existing facilities: very narrow wavelength coverage, observations usually not simultaneous for a wider spectral range with the introduction of systematic noise, insufficient time allocated to exoplanet science, and more in general the lack of a dedicated space-based exoplanet spectroscopy mission. Thus, our current knowledge of exoplanetary atmospheric and thermal characteristics is still very limited.

Ariel (Atmospheric Remote-sensing Infrared Exoplanet Large-survey) has been selected as the next ESA-M4 science mission [55] and it is expected to be launched in 2028. During its 4-year mission, Ariel will observe the atmospheres of a statistically representative sample $(\sim 1000)$ of transiting gaseous (Jupiters, Saturns, Neptunes) and rocky (super-Earths and sub-Neptunes) planets using transit spectroscopy in the $1.10-7.8 \mu \mathrm{m}$ spectral range and three narrow-bands photometry in the optical. The wavelength range proposed covers all the expected major atmospheric gases from $\mathrm{H}_{2} \mathrm{O}, \mathrm{CO}_{2}, \mathrm{CH}_{4}, \mathrm{NH}_{3}, \mathrm{HCN}, \mathrm{H}_{2} \mathrm{~S}$ up to the more exotic metallic compounds, such as $\mathrm{TiO}, \mathrm{VO}$, and condensed species. Ariel is designed as a dedicated survey mission for transit, eclipse and phase-curved spectroscopy, providing a homogeneous dataset, with a consistent pipeline and an well-defined target selection strategy, maximising the scientific yield. Focusing on transit, eclipse spectroscopy, the methods are based on the differential analysis of the star and planet spectra in and out of transit, allowing 
to measure planetary atmospheric signals of $10-100 \mathrm{ppm}$ relative to the star. Such small signals require an exact knowledge of the host star spectrum, at least at the same level of the planetary signal, to map any stellar intrinsic variation (i.e. due to magnetic activity and convective turbulence) in order to avoid misleading results with planetary features [39].

Another important point is that information on the host star composition is critical to separate the signatures left on the planet by its formation, evolution and migration processes, from those due to the specific chemistry of the host star [63]. Indeed, recent studies suggest that planetary $\mathrm{O} / \mathrm{H}, \mathrm{C} / \mathrm{H}, \mathrm{C} / \mathrm{O}$ ratios and metallicity with respect to the stellar values could provide stronger constraints on the planet formation region and their migration mechanisms (see [27] and references therein for a recent review), but similar considerations apply to other elements (e.g., N, S, Ti, Al, [63]).

Finally, an increasing number of studies have pointed towards the existence of correlations between the properties of the host stars and the characteristics and frequency of their planetary systems. In this respect, the correlation between the stellar metallicity and the frequency of giant planets [41, 49, 64], the connection between radius vs metallicity $[13,43]$, eccentricity vs metallicity $[3,67]$, the role of the abundances of other elements $[4,16,17]$ in the host stars are only few examples of different results that take a clear shape as the new planet discoveries increase, shading light on many details still missing concerning planet formation and evolution.

Such works rely upon homogeneously and precisely derived stellar parameters. Therefore, homogeneous derivation of stellar parameters using high-quality data is crucial for characterising exoplanet-host stars [5, 42, 47], and in turn, is fundamental to improve the accuracy of the planet properties.

It is important to underline how a well-defined target selection strategy and the definition of the input target list with a statistically significant dataset in the range of relevant stellar/planet parameters have a fundamental role for maximising the scientific yield and the achievement of scientific goals of Ariel. A meaningful choice of the targets requires an accurate study of the stellar properties that need to be derived in advance and continuously updated as the mission approaches launch and the target list evolves with the new exoplanet discoveries.

In this context, we have started a benchmarking analysis between three different spectroscopic techniques used to determine stellar parameters for selected targets belonging to the Ariel Reference Sample [19]. Our goal is to consolidate a method that will be applied to homogeneously determine the stellar parameters for the complete Ariel Reference Sample. More generally, we refer also to the works of [26] and [45] where comparisons between the results from different spectroscopic techniques are discussed.

For a global approach to characterize the stars of Ariel Reference Sample see also [15], where an overview on the methods used to determine stellar fundamental parameters, elemental abundances, activity indices, and stellar ages for the Ariel Reference Sample is given and in particular, results for the homogeneous estimation of elemental abundances of $\mathrm{Al}, \mathrm{Mg}, \mathrm{Si}, \mathrm{C}, \mathrm{N}$, and the activity indices $\mathrm{S}$ and $\log \left(\mathrm{R}^{\prime} \mathrm{HK}\right)$ are presented. 
In the following sections we describe the star sample analysed, we give an overview of the methodology applied to derive the stellar parameters and then we present a comparative analysis of the results.

\section{Star sample}

On the bases of Ariel capabilities, a list of targets (Ariel Reference Sample) to be observed during the primary mission life was prepared for the Phase A [19, 69]. The sample includes $\sim 1000$ potential targets with stellar types FGKM (typically brighter than $\mathrm{K}=11 \mathrm{mag}$ ) and planetary parameters in a range of size between Jupiter down to Earth-like planets, temperature in the range between $500 \mathrm{~K}-2500 \mathrm{~K}$ and bulk density between $0.10-10.0 \mathrm{~g} / \mathrm{cm}^{3}$.

We started our analysis cross-matching the Ariel Reference Sample with the stars available in the SWEET-Cat (Stars With ExoplanETs Catalogue; https://www.astro. up.pt/resources/sweet-cat/) and we selected all the common targets with parameters source "flag=1", which are the stars analysed homogeneously (see [42]). Thus, our starting sub-sample includes $155 \mathrm{FGK}$ stars in a range of $5<\mathrm{V}$ (mag) $<16$ with relative spectra having a signal-to-noise ratio $(\mathrm{S} / \mathrm{N})$ between $\sim 50$ and $\sim 800$ (see Fig. 1). Stellar parameters for these 155 stars available in the SWEET-Cat, were considered our baseline for the comparison analysis. An accurate description of the spectroscopic data used to derive SWEET-Cat stellar parameters is presented in [47] and [42].

We then re-determined the stellar parameters of this sub-sample using two different methods: FAMA (Fast Automatic MOOG Analysis, [28]), based on the equivalent widths analysis and FASMA (Fast Analysis of Spectra Made Automatically, [59]), based on the spectral synthesis method. For our work, we used the same archival spectra employed for the SWEET-Cat analysis. The characteristics of each spectrograph and the number of stars considered in our analysis for each instrument are listed in Table 1. Excluding stars for which FAMA and FASMA codes did not converge (spectra dominated by fringing, very low $\mathrm{S} / \mathrm{N}$, fast rotator targets, very cool stars), we obtained results for about $93 \%$ of the sample.

Individual Stellar parameters derived by FAMA and FASMA for each analysed star are provided as online material in the format as Table 2.

\section{Derivation of stellar parameters}

\subsection{SWEET-Cat}

SWEET-Cat is a catalog of stellar parameters taken in general from the literature for planet hosting stars listed within the Extrasolar Planets Encyclopaedia (http://exoplanets.eu/). This catalogue is continuously updated when new planets are announced and new stellar parameters derived. In particular, for stars with spectra acquired with high resolution spectrographs (mainly HARPS, UVES, FEROS) and high signal-to-noise ratios (mostly $\mathrm{S} / \mathrm{N}>100$ ), the stellar parameters are obtained in a homogeneous way (flagging the targets with 1 , as mentioned above). The method 

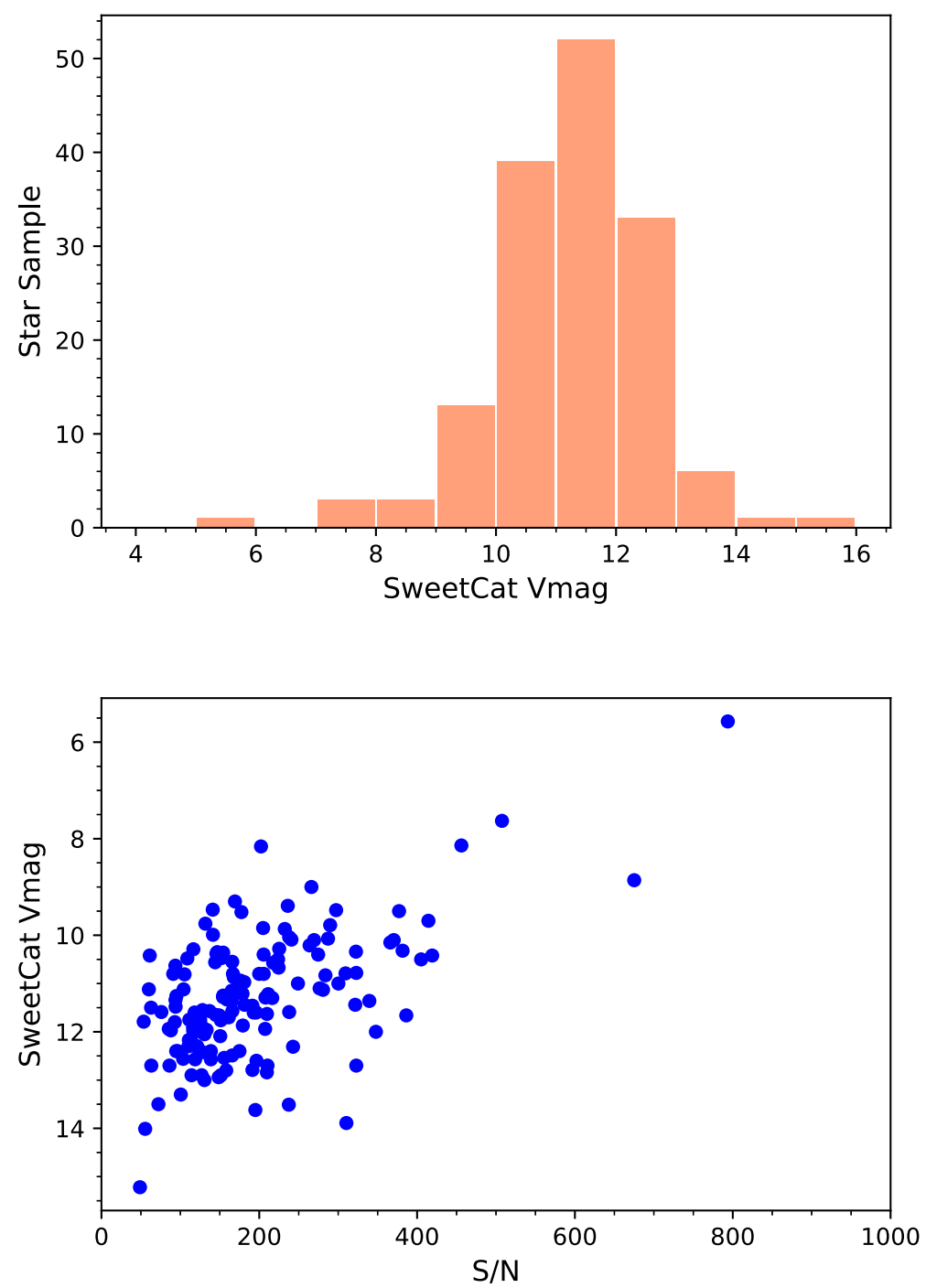

Fig. 1 Top panel: Distribution of the star sample analyzed as a function of V mag listed in the SWEET-Cat catalog. Bottom panel: V mag vs $\mathrm{S} / \mathrm{N}$ for our star sample

used to derive stellar parameters is described in, e.g., [41] and [49]. Briefly, local thermodynamic equilibrium (LTE) condition is assumed, a grid of Kurucz planeparallel model atmospheres ([25], ATLAS9), and the ARES code [48] for measuring line equivalent widths (EWs) are considered. Stellar parameters (effective temperature $\mathrm{T}_{\text {eff }}$, surface gravity $\log \mathrm{g}$, microturbulence velocity $\mathrm{V}_{\text {micro }}$, and iron abundance $[\mathrm{Fe} / \mathrm{H}]$ ) are derived using the MOOG code (version 2002; [46]) and the line list by [49]. Excitation and ionization equilibria of the Fe I and Fe II weak lines were 
Table 1 Spectrograph information: resolving power, spectral ranges and number of stars considered

\begin{tabular}{llll}
\hline Instrument & $\begin{array}{l}\text { Resolving Power } \\
(\lambda / \Delta \lambda)\end{array}$ & $\begin{array}{l}\text { Spectral Range } \\
(\AA)\end{array}$ & $\begin{array}{l}\text { N } \\
\text { of Stars }\end{array}$ \\
\hline ESPADONS & 80000 & $3700-10500$ & 2 \\
FEROS & 48000 & $3600-9200$ & 38 \\
FIES & 67000 & $3700-7300$ & 7 \\
HARPS & 100000 & $3800-7000$ & 39 \\
HARPSN & 115000 & $3830-6900$ & 4 \\
NARVAL & 80000 & $3700-10500$ & 2 \\
SARG & $57000-86000$ & $5100-10100$ & 5 \\
SOPHIE & 75000 & $3820-6920$ & 15 \\
UVES & 110000 & $3000-6800$ & 43 \\
\hline
\end{tabular}

imposed to derive stellar parameters. The errors on the atmospheric parameters were derived as in [41] and references therein.

The stellar masses listed in the SWEET-Cat catalog were derived with the calibration presented in [56], using as input the spectroscopic parameters. According to [42], a correction was applied for the cases in which the calibration gives values between 0.7 and $1.3 M_{\odot}$. The errors for these mass values are computed as in [42] by means of a Monte Carlo analysis. For each case 10000 random values of effective temperature, surface gravity, and stellar metallicity were drawn from a Gaussian distribution. From the resulting mass distribution, the central value for the mass and 1 -sigma uncertainty were derived.

\subsection{FAMA analysis}

The aim of FAMA is to allow the computation of the atmospheric parameters and abundances of a large number of stars using measurements of equivalent widths (EWs) as automatic and as independent of any subjective approach as possible. It is based on the simultaneous search for three equilibria: excitation equilibrium, ionization balance, and the relationship between $\log n(\mathrm{FeI})$ and EW/ $\lambda$. FAMA also evaluates the statistical errors on individual element abundances and errors due to the uncertainties in the stellar parameters. The convergence criteria are not fixed a priori but are based on the quality of the spectra. The code is described in [28]. For our work, first, we have measured the EWs with DOOp (DAOSPEC Option Optimiser, [14]) an automatic tool developed within the Gaia-ESO survey. The code is based on DAOSPEC code [53] and uses Gaussian fit to measure EWs. We adopted the line list of the Gaia-ESO survey [23], which includes atomic parameters (log $g$ and damping coefficient) for a large number of lines in the spectral range 4200-6800 $\AA$. We adopted the MARCS model atmospheres (plane parallel and spherical) [22]. 


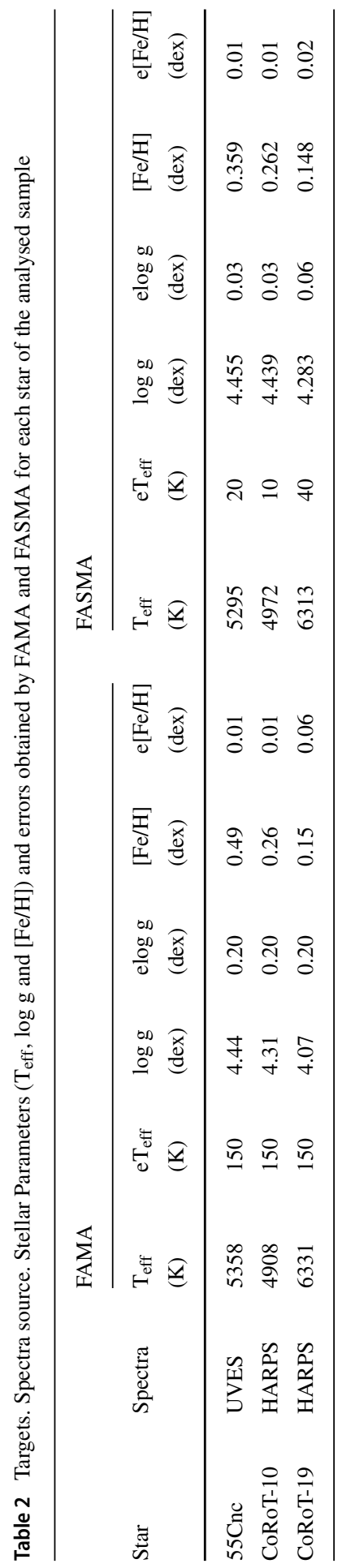




\subsection{FASMA analysis}

The analysis with FASMA is based on the spectral synthesis technique wrapped around the radiative transfer code, MOOG (version 2019; [46]).

FASMA creates synthetic spectra on-the-fly and delivers the best-fit parameters (effective temperature, surface gravity, iron abundance, and projected rotational velocity) after a non-linear least-squares fit (Levenberg-Marquardt algorithm). The line list is mainly comprised of iron lines initially obtained from VALD3 [40]. The regions of the spectral synthesis are defined within small intervals around these iron lines $( \pm 2 \AA)$. The atomic data are calibrated to match the spectra of the Sun and Arcturus but given the strongest constrains on the solar parameters, we gave higher weights to the Sun. The damping parameters are based on the ABO theory [7] when available, or in any other case, we use the Blackwell approximation. The model atmospheres are interpolated from the ATLAS grid [25] in LTE. The minimization is a two step procedure where initially we obtain the best-fit values using fixed solar macroand microturbulence. Then, we refine our results in a second step, with updated macro- and micro-turbulence based on empirical relations. Macroturbulence velocity is set by the calibration of [18] and microturbulence is set based on calibrations for either dwarfs [60] or giants [1]. The uncertainties are derived from the covariance matrix constructed by the nonlinear least-squares fit. The methodology is described in detail in $[58,59]$ where it is tested for both giant and dwarf samples, including stars with larger rotation.

\section{Comparison among the methods}

We compare our results seeking possible outliers and trends among the parameters obtained by the three methods. In this preliminary work, we explore the results of the three analysis, comparing with external benchmarks, as the surface gravities derived from light-curves in literature and from Gaia parallaxes and photometry, to identify the ranges of best performances.

\subsection{SWEET-Cat vs FAMA/FASMA: $\mathrm{T}_{\text {eff }}$}

Figure 2 (top panels) shows the difference in effective temperature $\left(\mathrm{T}_{\mathrm{eff}}\right)$ between SWEET-Cat and FAMA (left panel) or SWEET-Cat and FASMA (right panel) as a function of the SWEET-Cat $\mathrm{T}_{\text {eff }}$. The color scale represents $\log \mathrm{g}$ for SWEET-Cat.

The bulk of the $\mathrm{T}_{\mathrm{eff}}$ results for the SWEET-Cat and FAMA is in good agreement (within 1- $\sigma$ ), although there are some outliers: 28 stars show a difference in $\mathrm{T}_{\text {eff }}$ over $1 \sigma$ level but still within $\sim 417 \mathrm{~K}$ ( $3 \sigma$ level). Among these outliers, we noticed that 7 stars present large vsini $(>10 \mathrm{~km} / \mathrm{s})$. The $\mathrm{T}_{\text {eff }}$ spread seems more evident when considering stars with the lower and higher $\mathrm{T}_{\text {eff }}$ in the considered range. In addition, there is an offset between the two methods, with the mean value (red dashed central line) for the difference of $\sim 70 \mathrm{~K}$. 

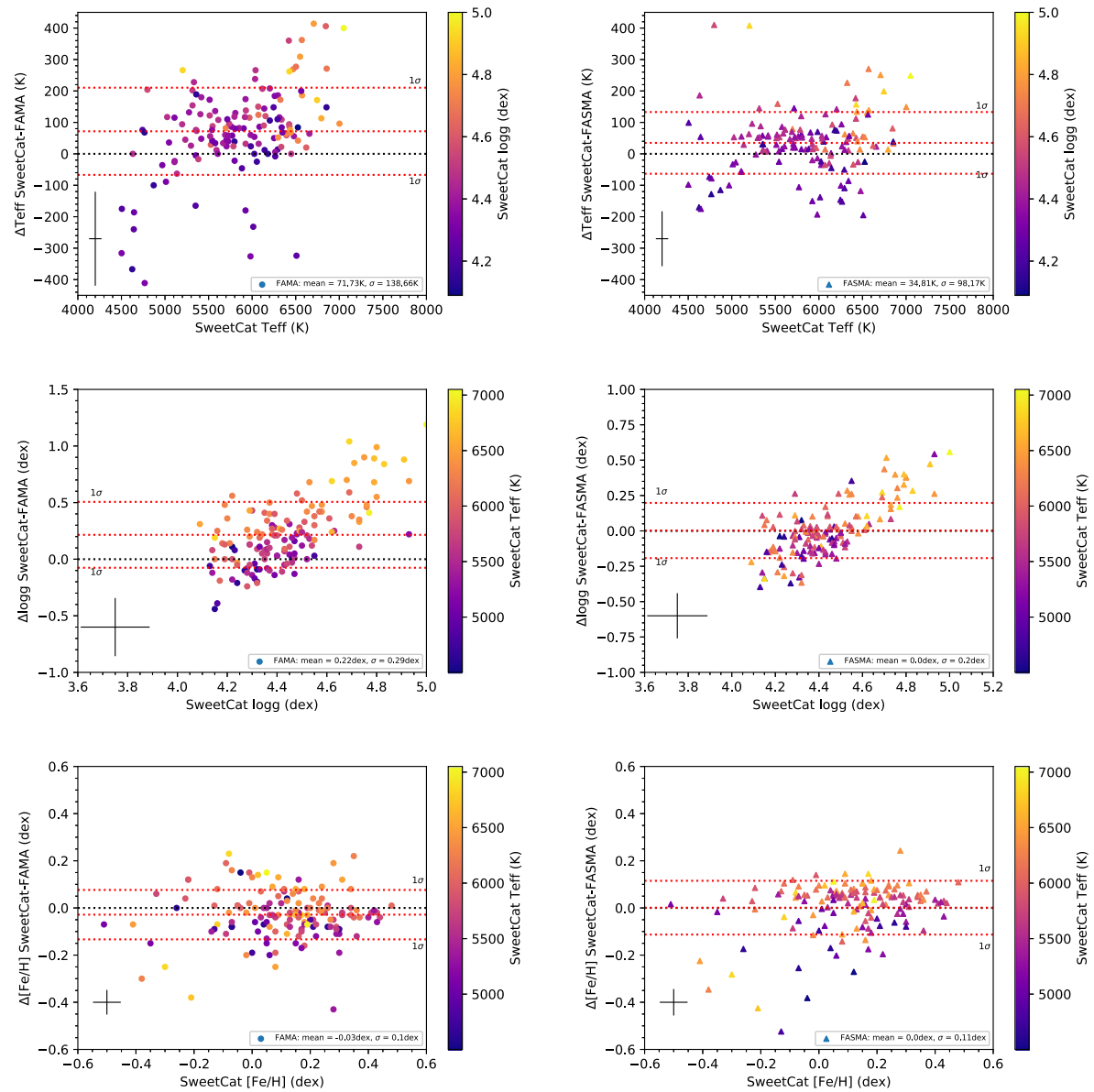

Fig. 2 Top panels: Difference between $\mathrm{T}_{\text {eff }}$ from SWEET-Cat and $\mathrm{T}_{\text {eff }}$ from FAMA (left)/FASMA (right) as a function of the $T_{\text {eff }}$ SWEET-Cat. The colour scale indicates the value of log $g$ for SWEET-Cat. Red dashed line: average of the $\mathrm{T}_{\text {eff }}$ difference and $1 \sigma$ level. Central panels: Difference between $\log \mathrm{g}$ from Sweet-Cat and log g from FAMA (left)/FASMA (right) as a function of log $g$ from SWEET-Cat.The colour scale indicates the value of $\mathrm{T}_{\text {eff }}$ for SWEET-Cat. Bottom panels: Difference between $[\mathrm{Fe} / \mathrm{H}]$ derived using SWEET-Cat and FAMA (left)/FASMA (right) as a function of [Fe/H] from SWEET-Cat. The colour scale represents $\mathrm{T}_{\mathrm{eff}}$ from SWEET-Cat. Average errors are also indicated on the left of each plot

Focusing now on the FASMA analysis, also in this case, the bulk of the $\mathrm{T}_{\text {eff }}$ results for the SWEET-Cat and FASMA is in good agreement (within $1 \sigma$ ), although outliers are also present. For stars with the lower and higher $\mathrm{T}_{\text {eff }}$ in the range of interest, the dispersion between the two methods increases. An offset between the two methods is present with a mean value (red dashed line) in the difference of $\sim 34 \mathrm{~K}$. We note here that some of the outliers are not in common with the outliers found using the FAMA method. 


\subsection{SWEET-Cat vs FAMA/FASMA: $\log \mathrm{g}$}

Figure 2 (central panels) show respectively the comparison of $\log g$ derived using the FAMA (left panel) or FASMA (right panel) methods vs the results given in SWEET-Cat. The colour scale represents $\mathrm{T}_{\text {eff }}$ for SWEET-Cat. There are trends in both the FAMA and FASMA results: the differences between their log $g$ and those of SWEET-cat are increasing with $\log \mathrm{g}$. The differences are particularly high for higher surface gravity ( $\log g>4.6 \mathrm{dex})$. In the log g range of $\sim 4-4.6 \mathrm{dex}$, the trend is almost negligible. In addition, FAMA, on average, gives lower gravities, of about $\sim-0.18$ dex.

\subsection{SWEET-Cat vs FAMA/FASMA: [Fe/H]}

Figure 2 (bottom panels) show respectively the comparison of $[\mathrm{Fe} / \mathrm{H}]$ derived using the FAMA (left panels) method and FASMA (right panels) vs the results given in SWEET-Cat. The colour scale represents $\mathrm{T}_{\mathrm{eff}}$ for SWEET-Cat.

Considering the typical errors on metallicity $(\sim 0.10 \mathrm{dex})$, the results are quite satisfactory: there are almost no trend in the differences vs $[\mathrm{Fe} / \mathrm{H}]$ and the means are close to zero (slightly negative for FAMA).

This is one of the most important results of the analysis and we need to remark it. Despite the differences in the derived stellar parameters, the three methods converge to very similar final metallicities $[\mathrm{Fe} / \mathrm{H}]$. The three methods rely, indeed, on different line lists, with different set of atomic data, which make them to converge on slightly different sets of $\mathrm{T}_{\text {eff }}$ and $\log \mathrm{g}$ values, which should satisfy the conditions of excitation equilibrium and ionization balance. $\mathrm{T}_{\mathrm{eff}}$ and $\log \mathrm{g}$ tend to vary along a local minimum, in which the value of $[\mathrm{Fe} / \mathrm{H}]$ is usually more constant.

In Fig. 3, we show the differences in metallicity of the FAMA and FASMA results with respect to SWEET-Cat, as a function of the differences in the derived surface gravities. The symbol are colour-codes by the differences in the derived $\mathrm{T}_{\text {eff }}$, again, with respect to the SWEET-Cat ones.
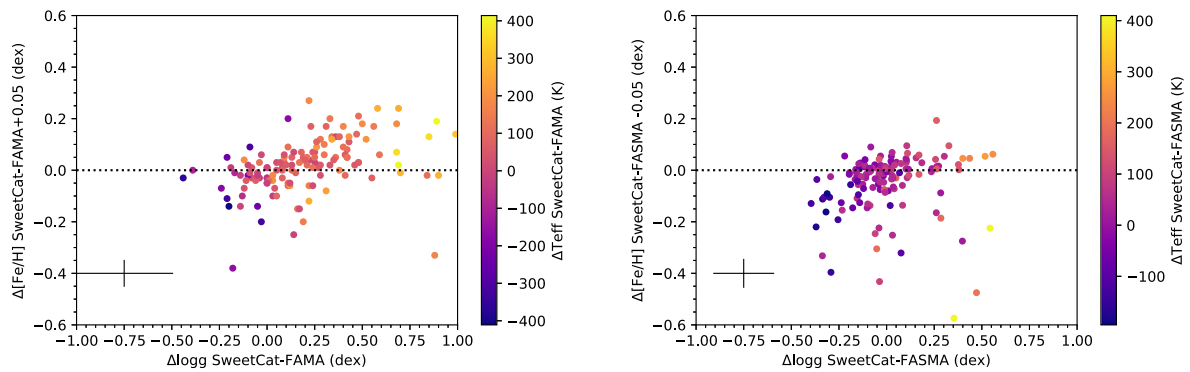

Fig. 3 Left panel: SWEET-Cat vs FAMA differences in $\log \mathrm{g}$ vs differences in $[\mathrm{Fe} / \mathrm{H}]$, colour-coded by differences in $\mathrm{T}_{\text {eff }}$. Right panel: the same plot for the FASMA results. An offset of +0.05 dex is applied to FAMA metallicities, while an offset of -0.05 dex to the FASMA ones 
Removing the systematic offsets (+0.05 dex from FAMA and -0.05 dex for FASMA with respect to the SWEET-Cat scale), the differences in $[\mathrm{Fe} / \mathrm{H}]$ are negligible for variation in the surface gravity within \pm 0.3 dex with respect to the SWEET-Cat ones. In the comparison between FAMA and SWEET-cat $[\mathrm{Fe} / \mathrm{H}]$ even for differences in $\log \mathrm{g} \sim-0.3 \mathrm{dex}$, the agreement in metallicity between the two methods is still good. Larger differences in $\log \mathrm{g}>+0.3 \mathrm{dex}$, corresponds to higher $[\mathrm{Fe} / \mathrm{H}]$ in SWEET-Cat than the ones obtained with the other methods. This means that moving to higher effective temperatures the discrepancies between the methods increases also for the results in $[\mathrm{Fe} / \mathrm{H}]$ and the hottest temperature regime remains thus critical also to obtain reliable metallicities.

Concluding, the stars in the parameter space closer to the solar values $\left(\mathrm{T}_{\text {eff }}=5000-\right.$ $6000 \mathrm{~K}, \log \mathrm{g}=4.2-4.6 \mathrm{dex})$ are those for which the three methods are in better agreement. Among the three parameters, the best accord is reached for $[\mathrm{Fe} / \mathrm{H}]$ in the whole parameter space. The most critical parameter is, however, the surface gravity: external comparison (using Gaia data, asteroseismic data, isochrones) are needed to evaluate the results of the different methods. A correct evaluation of all stellar parameters is indeed fundamental for a precise characterisation of the host star, including the determination of its age [9].

\section{Evaluation of the accuracy and precision of the stellar parameters}

In this section, we discuss some indirect checks on the accuracy and precision of the stellar parameters. We especially focus on the control of the stellar surface gravity, parameter that cannot be usually constrained well by spectroscopic methods (e.g. [30, $61])$. This fact has an impact on the calculation of other stellar parameters $\left(\mathrm{T}_{\mathrm{eff}}\right.$ and $[\mathrm{Fe} / \mathrm{H}])$ and subsequently on the derivation of the stellar chemical abundances.

\subsection{Comparison with surface gravity derived from trigonometric distances using Gaia DR2}

We compare the log g derived using the three spectroscopic methods with the trigonometric log g based on Gaia DR2 photometry and parallax. Photometric gravities have been obtained using the following equation

$$
\log (g)=\log \left(M / M_{\odot}\right)+0.4 \cdot M_{\text {bol }}+4 \cdot \log \left(T_{\text {eff }}\right)-12.505
$$

where $M / M_{\odot}$ is the stellar mass (in solar mass units) provided by the Sweet-cat database. $M_{\text {bol }}$ is the bolometric magnitude, obtained from the luminosity published in the Gaia DR2 catalog [21] using the following relation $\mathrm{M}_{\text {bol }}=4.75-2.5 \times \log \left(\mathrm{L} / \mathrm{L}_{\odot}\right)$, and $\mathrm{T}_{\mathrm{eff}}$ is the Gaia photometric $\mathrm{T}_{\mathrm{eff}}$. Since the stars in our sample are nearby and thus the reddening is negligible, we can derive $M_{b o l}$ from the Gaia luminosities and the distances obtained directly by inverting the parallaxes. In Fig. 4 (left top and bottom panels) we show the comparison (no outliers are removed in the plot). Surface gravities from the SWEET-Cat appear to be slightly underestimated at low $\mathrm{T}_{\text {eff }}$, and highly overestimated at high $\mathrm{T}_{\text {eff }}$. The same behaviour for FASMA at high $\mathrm{T}_{\text {eff }}$. No

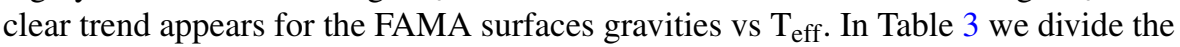



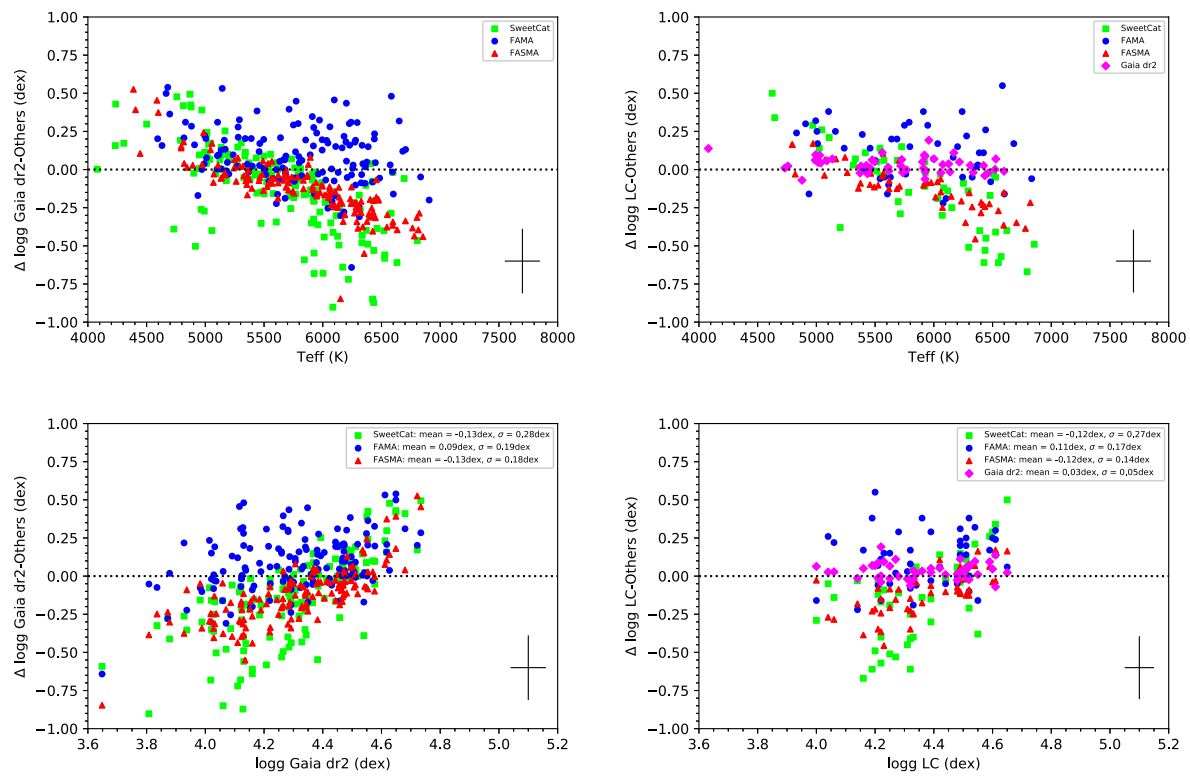

Fig. 4 Left panels: Comparison between spectroscopic (Sweet-cat, FAMA and FASMA) and trigonometric $\log g$ as a function of $\mathrm{T}_{\text {eff }}$ (top) and of trigonometric $\log \mathrm{g}$ (bottom). The corresponding $\mathrm{T}_{\text {eff }}$ for the trigonometric $\log \mathrm{g}$, is derived from the Gaia measurements. Right panels: Comparison between spectroscopic (Sweet-cat, FAMA and FASMA) and $\log g$ derived from the light-curves as a function of $\mathrm{T}_{\mathrm{eff}}$ (top) and of light-curve log g (bottom). Average errors are also indicate on a side of each plot. For the parameter differences we considered as error the larger value among the average errors from each method

sample in three temperature regimes: $\mathrm{T}_{\text {eff }}<5000 \mathrm{~K}, 5000 \mathrm{~K}<\mathrm{T}_{\text {eff }}<6000 \mathrm{~K}$, and finally $\mathrm{T}_{\text {eff }}>6000 \mathrm{~K}$. We compute the mean differences between the log $\mathrm{g}$ from Gaia and the spectroscopic log $\mathrm{g}$ from the three methods, and their standard deviation (1$\sigma)$. In the coolest regime, all methods tend to underestimate the surface gravities, with differences consistent with each other within the error. The spectral synthesis method FASMA provides gravities, on average, in better agreement with the trigonometric ones. In the intermediate regime, with $5000 \mathrm{~K}<\mathrm{T}_{\text {eff }}<6000 \mathrm{~K}$, the three methods show the better agreement with the log g from Gaia: FAMA slightly underestimates

Table 3 Mean differences $\log \mathrm{g}_{\text {Gaia }}-\log \mathrm{g}_{\text {spec }}$ with standard deviation $(1-\sigma)$ and median differences (in parenthesis) in three $\mathrm{T}_{\text {eff }}$ intervals

\begin{tabular}{lllll}
\hline Method & $\begin{array}{l}\mathrm{T}_{\text {eff }}<5000 \\
\mathrm{~K}\end{array}$ & $\begin{array}{l}5000 \mathrm{~K}<\mathrm{T}_{\text {eff }}< \\
6000 \mathrm{~K}\end{array}$ & $\begin{array}{l}\mathrm{T}_{\text {eff }}>6000 \\
\mathrm{~K}\end{array}$ & $\begin{array}{l}\text { Total } \\
\text { range }\end{array}$ \\
\hline SWEET-Cat & $0.132 \pm 0.068$ & $-0.042 \pm 0.017$ & $-0.381 \pm 0.037$ & $-0.115 \pm 0.023$ \\
& $(0.165)$ & $(-0.032)$ & $(-0.393)$ & $(-0.083)$ \\
FAMA & $0.162 \pm 0.043$ & $0.059 \pm 0.020$ & $0.108 \pm 0.029$ & $0.088 \pm 0.016$ \\
& $(0.160)$ & $(0.053)$ & $(0.126)$ & $(0.064)$ \\
FASMA & $0.088 \pm 0.051$ & $-0.086 \pm 0.011$ & $-0.280 \pm 0.014$ & $-0.137 \pm 0.013$ \\
& $(0.037)$ & $(-0.084)$ & $(-0.289)$ & $(-0.116)$ \\
\hline
\end{tabular}


the trigonometric gravities, while SWEET-Cat and FASMA slightly overestimate them. The most challenging regime is the hottest one, with $\mathrm{T}_{\text {eff }}>6000 \mathrm{~K}$. In this temperature range, there is no agreement between the spectroscopic methods and the trigonometric one (FASMA and SWEET-Cat overestimate the trigonometric log g, while FAMA slightly underestimates it). In the last column, we report the mean differences for the whole sample: slightly negative differences for SWEET-cat and FASMA, and positive for FAMA. However, it is clear that global mean differences mask the most critical regimes, for the hottest and coolest stars of the sample.

\subsection{Comparison with log g derived from the light-curves}

We compare the trigonometric and spectroscopic gravities with those derived from the light-curves in literature, and available in the SWEET-Cat database. In Fig. 4 (right top and bottom panels) we show the difference between the log g derived from the light-curves (literature values for 48 targets were found) and the respective spectroscopic log g listed in the SWEET-Cat (green filled squares) and obtained through FASMA (red filled triangles) and FAMA (blue filled circles) as a function of $\mathrm{T}_{\text {eff }}$ and of the light-curves $\log$ g. First, we notice the good agreement between the trigonometric and light-curve surface gravities, despite the two methodologies are quite different, and this is an encouraging result (see also Fig. 5). Concerning the other methods, similar considerations as in the previous section can be obtained: FAMA shows a bit higher dispersion and a positive offset, but almost no trend. FASMA displays the lower dispersion, but tends to overestimate $\log g$ at high $\mathrm{T}_{\text {eff. }}$ SWEET-cat seems to overestimate $\log \mathrm{g}$ at high $\mathrm{T}_{\text {eff }}$, and underestimate at low $\mathrm{T}_{\text {eff }}$. Clearly the hottest region is the most critical one, both because hot stars have less absorption lines useful to constrain the photospheric parameters and because stellar rotation might

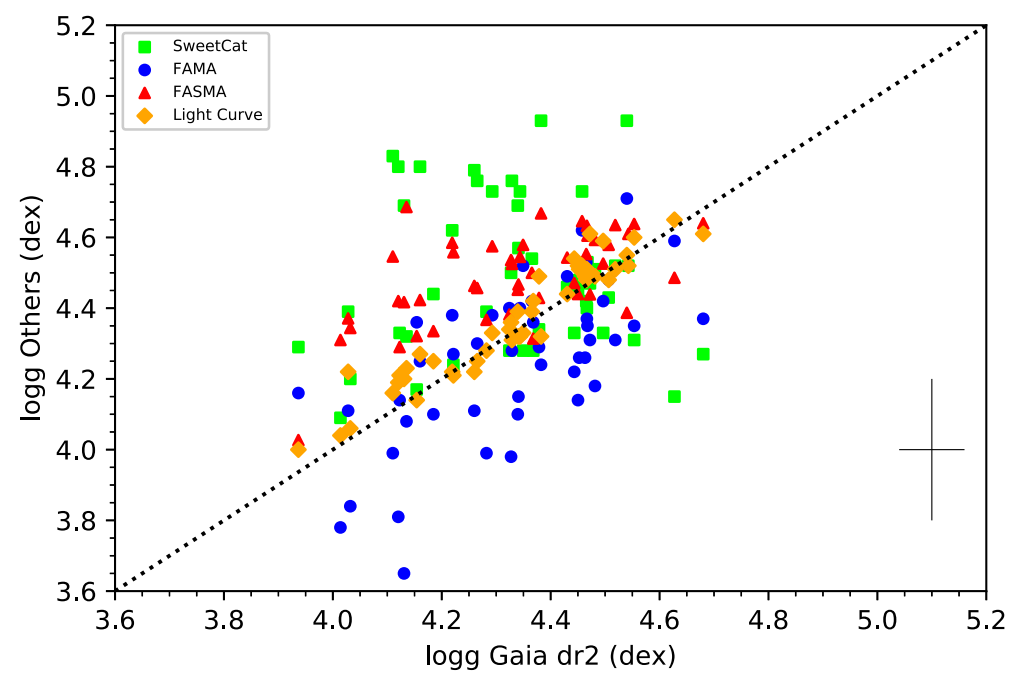

Fig. 5 Trigonometric surface gravity vs surface gravity derived with the other methods, including spectroscopic methods and light-curve method 

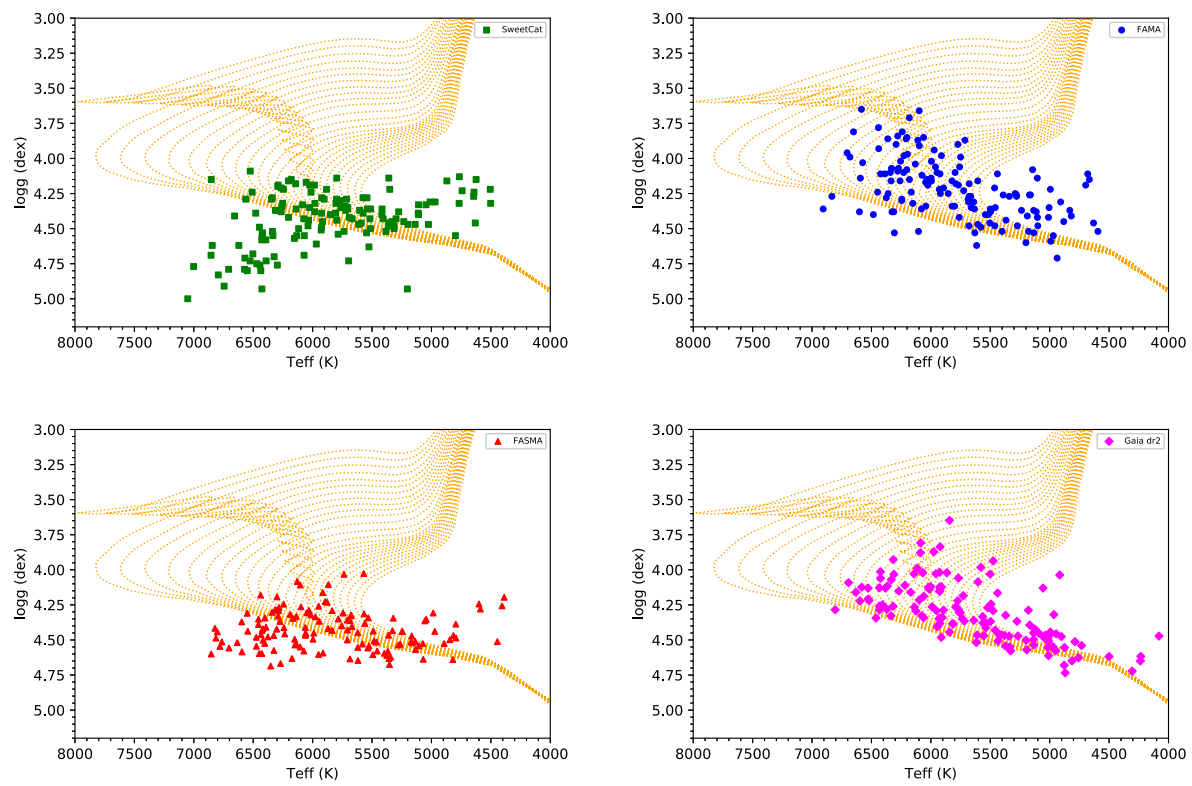

Fig. 6 Kiel diagram ( $\log g$ vs $T_{\text {eff }}$ ) using values listed in SWEET-Cat, obtained by FAMA, FASMA and derived by Gaia. The outermost tracks correspond to $\log ($ age/yr) $=8.95$ (bottom track) and 10.15 (upper track) with $[\mathrm{M} / \mathrm{H}]=0.058, Z=0.0198, Y=0.273$

become important, making more difficult to measure absorption lines which can be blended. Moreover, in the low-temperature regime, the presence of molecular bands can blend and hide the atomic lines.

\subsection{Comparison with isochrones}

Another important check is based on the comparison with theoretical isochrones, computed in $\mathrm{T}_{\text {eff }}$ vs $\log \mathrm{g}$ plane, for a set of ages, keeping the metallicity constant at $[\mathrm{M} / \mathrm{H}]=0.058 .{ }^{1}$ Figure 6 shows the Kiel diagrams (log g vs $\left.\mathrm{T}_{\text {eff }}\right)$ for the values of $\log \mathrm{g}$ and $\mathrm{T}_{\text {eff }}$ listed in the Sweet-Cat, obtained by FAMA and by FASMA, and derived through Gaia photometry and parallax, respectively. The PARSEC isochrones [10] in a range of ages are over-plotted: the outermost tracks correspond to $\log ($ age/yr $)=$ 8.95 (bottom track) and 10.15 (upper track) with $[\mathrm{M} / \mathrm{H}]=0.058, Z=0.0198, Y=$ 0.273 .

Figure 6 (left top panel) points out how the SWEET-Cat gravities are not matching the isochrones path and appear to be overestimated at the highest temperatures and underestimated at the lowest temperatures. In Fig. 6 (right top panel) we present the same plot for the results obtained with FAMA: $\mathrm{T}_{\text {eff }}$ and $\log \mathrm{g}$ follow the expected trend, however there is an offset towards lower gravities. In Fig. 6 (left bottom panel)

\footnotetext{
${ }^{1}$ Most of our targets have indeed close-to-Solar metallicity, with a mean $[\mathrm{Fe} / \mathrm{H}]$ of the sample $+0.11 \pm 0.18 \mathrm{dex}([\mathrm{Fe} / \mathrm{H}]$ from Sweet-cat values $)$
} 
we show the results of FASMA. The dispersion is lower, however, at high temperature there is still an overestimation of log g. Finally, in Fig. 6 (right bottom panel) the Gaia parameters are displayed, pointing out the best match with PARSEC isochrones.

\section{Conclusions}

In our first test, we have compared the analysis of a sample of $\sim 150$ spectra (high $\mathrm{S} / \mathrm{N}$ and high spectral resolution). In the parameters space close to Solar values the agreement among the three methods is good $\left(\mathrm{T}_{\text {eff }}=5000-6000 \mathrm{~K}, \log \mathrm{g}=4.2-4.6 \mathrm{dex}\right)$. However, at low and high temperatures some methods tend to under/overestimate the surface gravity log g. This might have important effects on the derived stellar abundances. External comparisons (using trigonometric $\log \mathrm{g}$, $\log \mathrm{g}$ from light-curves and from isochrones) confirm the trends. Corrections to these trends were already available (see, e.g, $[17,31]$ ). We plan in the next tests to apply them, using both corrections from asteroseismology and light-curves to provide more realistic gravites. In this context we refer also to the wider discussion present in literature on the disagreement between spectroscopic and evolutionary or photometric log g values (e.g. see $[52,57])$.

However, it is important to notice that, despite the differences in the derived photometric parameters, especially in $\log g$, the three spectroscopic methods agree very well on the final metallicity, except in the hottest temperature regime. Due to high quality of the Gaia photometry and parallax for the Ariel targets, a viable and welcome possibility is to adopt the surface gravity homogeneously derived from Gaia to compute chemical abundances.

Supplementary Information The online version contains supplementary material available at https://doi.org/10.1007/s10686-020-09695-4.

Acknowledgements We like to thank the anonymous referees for the fruitful comments on our paper. AB, MT, LM, MvS, GC acknowledge the funding from MIUR Premiale 2016: MITIC. The authors acknowledge the support of the ARIEL ASIINAF agreement n. 2018-22-HH.0. E.D.M., V.A. and S.G.S. acknowledge the support from Fundação para a Ciência e a Tecnologia (FCT) through national funds and from FEDER through COMPETE2020 by the following grants: UID/FIS/04434/2019, UIDB/04434/2020 and UIDP/04434/2020; PTDC/FIS-AST/32113/2017 POCI-01-0145-FEDER032113; PTDC/FIS-AST/28953/2017 and POCI-01-0145-FEDER-028953. https://www.overleaf.com/ project/5ee29f0469a39e00013326b1. E.D.M., V.A., S.G.S. also acknowledge the support from FCT through Investigador FCT contracts IF/00849/2015/CP1273/CT0003, IF/00650/2015/CP1273/CT0001, IF/00028/2014/CP1215/CT0002.

Funding Open Access funding provided by Istituto Nazionale di Astrofisica.

Open Access This article is licensed under a Creative Commons Attribution 4.0 International License, which permits use, sharing, adaptation, distribution and reproduction in any medium or format, as long as you give appropriate credit to the original author(s) and the source, provide a link to the Creative Commons licence, and indicate if changes were made. The images or other third party material in this article are included in the article's Creative Commons licence, unless indicated otherwise in a credit line to the material. If material is not included in the article's Creative Commons licence and your intended use is not permitted by statutory regulation or exceeds the permitted use, you will need to obtain permission directly from the copyright holder. To view a copy of this licence, visit http://creativecommons.org/licenses/by/4.0/. 


\section{References}

1. Adibekyan, V., Figueira, P., Santos, N.C., Sousa, S.G., Faria, J.P., Delgado-Mena, E., Oshagh, M., Tsantaki, M., Hakobyan, A.A., González Hernández, J.I., Suárez-Andrés, L., Israelian, G.: Identifying the best iron-peak and $\alpha$,-capture elements for chemical tagging: The impact of the number of lines on measured scatter. A\&A 583, A94 (2015). https://doi.org/10.1051/0004-6361/201527120

2. Adibekyan, V., Sousa, S.G., Santos, N.C.: Characterization of exoplanet-host stars. In: Campante, T.L., Santos, N.C., Monteiro M.J.P.F.G. (eds.) Asteroseismology and Exoplanets: Listening to the Stars and Searching for New Worlds, vol. 49, pp 225 (2018). https://doi.org/10.1007/978-3-31959315-9_12

3. Adibekyan, V.Z., Figueira, P., Santos, N.C., Mortier, A., Mordasini, C., Delgado Mena, E., Sousa, S.G., Correia, A.C.M., Israelian, G., Oshagh, M.: Orbital and physical properties of planets and their hosts: new insights on planet formation and evolution. A\&A 560, A51 (2013). https://doi.org/10.1051/0004-6361/201322551

4. Adibekyan, V.Z., Sousa, S.G., Santos, N.C., Delgado Mena, E., González Hernández, J.I., Israelian, G., Mayor, M., Khachatryan, G.: Chemical abundances of 1111 FGK stars from the HARPS GTO planet search program. Galactic stellar populations and planets. A\&A 545, A32 (2012). https://doi.org/10.1051/0004-6361/201219401

5. Andreasen, D.T., Sousa, S.G., Tsantaki, M., Teixeira, G.D.C., Mortier, A., Santos, N.C., SuárezAndrés, L., Delgado-Mena, E., Ferreira, A.C.S.: SWEET-Cat update and FASMA. A new minimization procedure for stellar parameters using high-quality spectra. A\&A 600, A69 (2017). https://doi.org/10.1051/0004-6361/201629967

6. Artigau, É., Kouach, D., Donati, J.F., Doyon, R., Delfosse, X., Baratchart, S., Lacombe, M., Moutou, C., Rabou, P., Parès, L.P., Micheau, Y., Thibault, S., Reshetov, V.A., Dubois, B., Hernandez, O., Vallée, P., Wang, S.Y., Dolon, F., Pepe, F.A., Bouchy, F., Striebig, N., Hénault, F., Loop, D., Saddlemyer, L., Barrick, G., Vermeulen, T., Dupieux, M., Hébrard, G., Boisse, I., Martioli, E., Alencar, S.H.P., do Nascimento, J.D., Figueira, P.: SPIRou: the near-infrared spectropolarimeter/high-precision velocimeter for the Canada-France-Hawaii telescope. In: Ground-based and Airborne Instrumentation for Astronomy V, Society of Photo-Optical Instrumentation Engineers (SPIE) Conference Series, vol. 9147, pp 914715 (2014). https://doi.org/10.1117/12.2055663

7. Barklem, P.S., Piskunov, N., O'Mara, B.J.: A list of data for the broadening of metallic lines by neutral hydrogen collisions. A\&A 142, 467-473 (2000). https://doi.org/10.1051/aas:2000167

8. Bean, J.L., Désert, J.M., Kabath, P., Stalder, B., Seager, S., Miller-Ricci Kempton, E., Berta, Z.K., Homeier, D., Walsh, S., Seifahrt, A.: The optical and near-infrared transmission spectrum of the super-earth GJ 1214b: Further evidence for a metal-rich atmosphere. ApJ 743(1), 92 (2011). https://doi.org/10.1088/0004-637X/743/1/92

9. Bossini, D., Rodrigues, T., Campante, T., Sacco, G.G., Danielski, C., Khan, S.E.A.: Homogeneous determination of stellar age in the ariel reference sample in prep (2020)

10. Bressan, A., Marigo, P., Girardi, L., Salasnich, B., Dal Cero, C., Rubele, S., Nanni, A.: PARSEC: stellar tracks and isochrones with the PAdova and TRieste stellar evolution code. MNRAS 427(1), 127-145 (2012). https://doi.org/10.1111/j.1365-2966.2012.21948.x

11. Broeg, C., Fortier, A., Ehrenreich, D., Alibert, Y., Baumjohann, W., Benz, W., Deleuil, M., Gillon, M., Ivanov, A., Liseau, R., Meyer, M., Oloffson, G., Pagano, I., Piotto, G., Pollacco, D., Queloz, D., Ragazzoni, R., Renotte, E., Steller, M., Thomas, N.: CHEOPS: A transit photometry mission for ESA's small mission programme. In: European Physical Journal Web of Conferences, European Physical Journal Web of Conferences, vol. 47, pp 03005 (2013). https://doi.org/10.1051/epjconf/20134703005

12. Brogi, M., Snellen, I.A.G., de Kok, R.J., Albrecht, S., Birkby, J., de Mooij, E.J.W.: The signature of orbital motion from the dayside of the planet $\tau$ Boötis b. Nature 486(7404), 502-504 (2012). https://doi.org/10.1038/nature11161

13. Buchhave, L.A., Bizzarro, M., Latham, D.W., Sasselov, D., Cochran, W.D., Endl, M., Isaacson, H., Juncher, D., Marcy, G.W.: Three regimes of extrasolar planet radius inferred from host star metallicities. Nature 509(7502), 593-595 (2014). https://doi.org/10.1038/nature13254

14. Cantat-Gaudin, T., Donati, P., Pancino, E., Bragaglia, A., Vallenari, A., Friel, E.D., Sordo, R., Jacobson, H.R., Magrini, L.: DOOp, an automated wrapper for DAOSPEC. A\&A 562, A10 (2014). https://doi.org/10.1051/0004-6361/201322533 
15. Danielski, C., Brucalassi, A., Benatti, S., Campante, T., Delgado-Mena, E., Rainer, M., Sacco, G., Adibekyan, V., Biazzo, K., Bossini, D., Bruno, G., Casali, G., Kabath, P.E.A.: The homogeneous characterisation of Ariel host stars (2020)

16. Delgado Mena, E., Israelian, G., González Hernández, J.I., Sousa, S.G., Mortier, A., Santos, N.C., Adibekyan, V.Z., Fernand es, J., Rebolo, R., Udry, S., Mayor, M.: Li depletion in solar analogues with exoplanets. Extending the sample. A\&A 562, A92 (2014). https://doi.org/10.1051/0004-6361/201321 493

17. Delgado Mena, E., Tsantaki, M., Adibekyan, V.Z., Sousa, S.G., Santos, N.C., González Hernández, J.I., Israelian, G.: Chemical abundances of 1111 FGK stars from the HARPS GTO planet search program. II. Cu, Zn, Sr, Y, Zr, Ba, Ce, Nd, and Eu. A\&A 606, A94 (2017). https://doi.org/10.1051/00046361/201730535

18. Doyle, A.P., Davies, G.R., Smalley, B., Chaplin, W.J., Elsworth, Y.: Determining stellar macroturbulence using asteroseismic rotational velocities from Kepler. MNRAS 444, 3592-3602 (2014). https://doi.org/10.1093/mnras/stu1692

19. Edwards, B., Mugnai, L., Tinetti, G., Pascale, E., Sarkar, S.: An updated study of potential targets for Ariel. AJ 157(6), 242 (2019). https://doi.org/10.3847/1538-3881/ab1cb9

20. Fu, G., Deming, D., Knutson, H., Madhusudhan, N., Mandell, A., Fraine, J.: Statistical analysis of hubble/WFC3 transit spectroscopy of extrasolar planets. ApJL 847(2), L22 (2017). https://doi.org/10.3847/2041-8213/aa8e40

21. Gaia Collaboration, Brown, A.G.A., Vallenari, A., Prusti, T., de Bruijne, J.H.J., Babusiaux, C., BailerJones, C.A.L., Biermann, M., Evans, D.W., Eyer, L., Jansen, F., Jordi, C., Klioner, S.A., Lammers, U., Lindegren, L., Luri, X., Mignard, F., Panem, C., Pourbaix, D., Randich, S., Sartoretti, P., Siddiqui, H.I., Soubiran, C., van Leeuwen, F., Walton, N.A., Arenou, F., Bastian, U., Cropper, M., Drimmel, R., Katz, D., Lattanzi, M.G., Bakker, J., Cacciari, C., Castañeda, J., Chaoul, L., Cheek, N., De Angeli, F., Fabricius, C., Guerra, R., Holl, B., Masana, E., Messineo, R., Mowlavi, N., Nienartowicz, K., Panuzzo, P., Portell, J., Riello, M., Seabroke, G.M., Tanga, P., Thévenin, F., Gracia-Abril, G., Comoretto, G., Garcia-Reinaldos, M., Teyssier, D., Altmann, M., Andrae, R., Audard, M., Bellas-Velidis, I., Benson, K., Berthier, J., Blomme, R., Burgess, P., Busso, G., Carry, B., Cellino, A., Clementini, G., Clotet, M., Creevey, O., Davidson, M., De Ridder, J., Delchambre, L., Dell'Oro, A., Ducourant, C., Fernández-Hernández, J., Fouesneau, M., Frémat, Y., Galluccio, L., García-Torres, M., GonzálezNúñez, J., González-Vidal, J.J., Gosset, E., Guy, L.P., Halbwachs, J.L., Hambly, N.C., Harrison, D.L., Hernández, J., Hestroffer, D., Hodgkin, S.T., Hutton, A., Jasniewicz, G., Jean-Antoine-Piccolo, A., Jordan, S., Korn, A.J., Krone-Martins, A., Lanzafame, A.C., Lebzelter, T., Löffler, W., Manteiga, M., Marrese, P.M., Martín-Fleitas, J.M., Moitinho, A., Mora, A., Muinonen, K., Osinde, J., Pancino, E., Pauwels, T., Petit, J.M., Recio-Blanco, A., Richards, P.J., Rimoldini, L., Robin, A.C., Sarro, L.M., Siopis, C., Smith, M., Sozzetti, A., Süveges, M., Torra, J., van Reeven, W., Abbas, U., Abreu Aramburu, A., Accart, S., Aerts, C., Altavilla, G., Álvarez, M.A., Alvarez, R., Alves, J., Anderson, R.I., Andrei, A.H., Anglada Varela, E., Antiche, E., Antoja, T., Arcay, B., Astraatmadja, T.L., Bach, N., Baker, S.G., Balaguer-Núñez, L., Balm, P., Barache, C., Barata, C., Barbato, D., Barblan, F., Barklem, P.S., Barrado, D., Barros, M., Barstow, M.A., Bartholomé Muñoz, S., Bassilana, J.L., Becciani, U., Bellazzini, M., Berihuete, A., Bertone, S., Bianchi, L., Bienaymé, O., Blanco-Cuaresma, S., Boch, T., Boeche, C., Bombrun, A., Borrachero, R., Bossini, D., Bouquillon, S., Bourda, G., Bragaglia, A., Bramante, L., Breddels, M.A., Bressan, A., Brouillet, N., Brüsemeister, T., Brugaletta, E., Bucciarelli, B., Burlacu, A., Busonero, D., Butkevich, A.G. Buzzi, R., Caffau, E., Cancelliere, R., Cannizzaro, G., Cantat-Gaudin, T., Carballo, R., Carlucci, T., Carrasco, J.M., Casamiquela, L., Castellani, M., CastroGinard, A., Charlot, P. Chemin, L., Chiavassa, A., Cocozza, G., Costigan, G., Cowell, S., Crifo, F., Crosta, M., Crowley, C. Cuypers, J., Dafonte, C., Damerdji, Y., Dapergolas, A., David, P., David, M., de Laverny, P., De Luise, F. De March, R., de Martino, D., de Souza, R., de Torres, A., Debosscher, J., del Pozo, E., Delbo, M., Delgado, A., Delgado, H.E., Di Matteo, P., Diakite, S. Diener, C., Distefano, E., Dolding, C., Drazinos, P. Durán, J., Edvardsson, B., Enke, H., Eriksson, K. Esquej, P., Eynard Bontemps, G. Fabre, C., Fabrizio, M., Faigler, S., Falcão, A.J., Farràs Casas, M., Federici, L., Fedorets, G., Fernique, P., Figueras, F., Filippi, F., Findeisen, K., Fonti, A., Fraile, E., Fraser, M., Frézouls, B., Gai, M., Galleti, S., Garabato, D., García-Sedano, F., Garofalo, A., Garralda, N., Gavel, A., Gavras, P., Gerssen, J., Geyer, R., Giacobbe, P., Gilmore, G., Girona, S., Giuffrida, G., Glass, F., Gomes, M., Granvik, M., Gueguen, A., Guerrier, A., Guiraud, J., Gutiérrez-Sánchez, R., Haigron, R., Hatzidimitriou, D., Hauser, M., Haywood, M., Heiter, U., Helmi, A., Heu, J., Hilger, T., Hobbs, D., Hofmann, W., Holland, G., Huckle, H. E., Hypki, A., Icardi, V., Janßen, K., Jevardat de Fombelle, G., Jonker, P.G., Juhász, Á.L., Julbe, F., Karampelas, A., Kewley, A., Klar, J., Kochoska, 
A., Kohley, R., Kolenberg, K., Kontizas, M., Kontizas, E., Koposov, S.E., Kordopatis, G., KostrzewaRutkowska, Z., Koubsky, P., Lambert, S., Lanza, A.F., Lasne, Y., Lavigne, J.B., Le Fustec, Y., Le Poncin-Lafitte, C., Lebreton, Y., Leccia, S., Leclerc, N., Lecoeur-Taibi, I., Lenhardt, H., Leroux, F., Liao, S., Licata, E., Lindstrøm, H.E.P., Lister, T.A., Livanou, E., Lobel A., López, M., Managau, S., Mann, R.G., Mantelet, G., Marchal, O., Marchant, J.M., Marconi, M., Marinoni, S., Marschalkó, G., Marshall, D.J., Martino, M., Marton, G., Mary, N., Massari, D., Matijevič, G., Mazeh, T., McMillan, P.J., Messina, S., Michalik, D., Millar, N.R., Molina, D., Molinaro, R., Molnár, L., Montegriffo, P., Mor, R., Morbidelli, R., Morel, T., Morris, D., Mulone, A.F., Muraveva, T., Musella, I., Nelemans, G., Nicastro, L., Noval, L., O’Mullane, W., Ordénovic, C., Ordóñez-Blanco, D., Osborne, P., Pagani, C., Pagano, I., Pailler, F., Palacin, H., Palaversa, L., Panahi, A., Pawlak, M., Piersimoni, A.M., Pineau, F.X., Plachy, E., Plum, G., Poggio, E., Poujoulet, E., Prša, A., Pulone, L., Racero, E., Ragaini, S., Rambaux, N., Ramos-Lerate, M., Regibo, S., Reylé, C., Riclet, F., Ripepi, V., Riva, A., Rivard, A., Rixon, G., Roegiers, T., Roelens, M., Romero-Gómez, M., Rowell, N., Royer, F., Ruiz-Dern, L., Sadowski, G., Sagristá Sellés, T., Sahlmann, J., Salgado, J., Salguero, E., Sanna, N., Santana-Ros, T., Sarasso, M., Savietto, H., Schultheis, M., Sciacca, E., Segol, M., Segovia, J.C., Ségransan, D., Shih, I.C., Siltala, L., Silva, A.F., Smart, R.L., Smith, K.W., Solano, E., Solitro, F., Sordo, R., Soria Nieto, S., Souchay, J., Spagna, A., Spoto, F., Stampa, U., Steele, I.A., Steidelmüller, H., Stephenson, C.A., Stoev, H., Suess, F.F., Surdej, J., Szabados, L., Szegedi-Elek, E., Tapiador, D., Taris, F., Tauran, G., Taylor, M.B., Teixeira, R., Terrett, D., Teyssand ier, P., Thuillot, W., Titarenko, A., Torra Clotet, F., Turon, C., Ulla, A., Utrilla, E., Uzzi, S., Vaillant, M., Valentini, G., Valette, V., van Elteren, A., Van Hemelryck, E., van Leeuwen, M., Vaschetto, M., Vecchiato, A., Veljanoski, J., Viala, Y., Vicente, D., Vogt, S., von Essen, C., Voss, H., Votruba, V., Voutsinas, S., Walmsley, G., Weiler, M., Wertz, O., Wevers, T., Wyrzykowski, Ł., Yoldas, A., Žerjal, M., Ziaeepour, H., Zorec, J., Zschocke, S., Zucker, S., Zurbach, C., Zwitter, T.: Gaia data release 2. Summary of the contents and survey properties. A\&A 616, A1 (2018). https://doi.org/10.1051/0004-6361/201833051

22. Gustafsson, B., Edvardsson, B., Eriksson, K., Jørgensen, U.G., Nordlund, A., Plez, B.: A grid of MARCS model atmospheres for late-type stars. I. Methods and general properties. A\&A 486, 951-970 (2008). https://doi.org/10.1051/0004-6361:200809724

23. Heiter, U., Lind, K., Asplund, M., Barklem, P.S., Bergemann, M., Magrini, L., Masseron, T., Mikolaitis, Š., Pickering, J.C., Ruffoni, M.P.: Atomic and molecular data for optical stellar spectroscopy. Phys. 90(5), 054010 (2015). https://doi.org/10.1088/0031-8949/90/5/054010

24. Jehin, E., Gillon, M., Opitom, C., Manfroid, J., Hutsemékers, D., Magain, P.: TRAPPIST (transiting planets and planetesimals small telescope). In: European Planetary Science Congress, EPSC, pp 2013968 (2013)

25. Kurucz, R.: ATLAS 9 stellar atmosphere programs and $2 \mathrm{~km} / \mathrm{s}$ grid. ATLAS 9 stellar atmosphere programs and 2 km/s grid. Kurucz CD-ROM No. 13. Camb. Mass Smithson. Astrophys. Obs. 1993, 13 (1993)

26. Lebzelter, T., Heiter, U., Abia, C., Eriksson, K., Ireland, M., Neilson, H., Nowotny, W., Maldonado, J., Merle, T., Peterson, R., Plez, B., Short, C.I., Wahlgren, G.M., Worley, C., Aringer, B., Bladh, S., de Laverny, P., Goswami, A., Mora, A., Norris, R.P., Recio-Blanco, A., Scholz, M., Thévenin, F., Tsuji, T., Kordopatis, G., Montesinos, B., Wing, R.F.: Comparative modelling of the spectra of cool giants $\star \star$. A\&A 547, A108 (2012). https://doi.org/10.1051/0004-6361/201219142

27. Madhusudhan, N., Agúndez, M., Moses, J.I., Hu, Y.: Exoplanetary atmospheres-chemistry, formation conditions, and habitability. SSR 205(1-4), 285-348 (2016). https://doi.org/10.1007/s11214-0160254-3

28. Magrini, L., Randich, S., Friel, E., Spina, L., Jacobson, H., Cantat-Gaudin, T., Donati, P., Baglioni, R., Maiorca, E., Bragaglia, A., Sordo, R., Vallenari, A.: FAMA: An automatic code for stellar parameter and abundance determination. A\&A 558, A38 (2013). https://doi.org/10.1051/0004-6361/201321844

29. Mayor, M., Pepe, F., Queloz, D., Bouchy, F., Rupprecht, G., Lo Curto, G., Avila, G., Benz, W., Bertaux, J.L., Bonfils, X., Dall, T., Dekker, H., Delabre, B., Eckert, W., Fleury, M., Gilliotte, A., Gojak, D., Guzman, J.C., Kohler, D., Lizon, J.L., Longinotti, A., Lovis, C., Megevand, D., Pasquini, L., Reyes, J., Sivan, J.P., Sosnowska, D., Soto, R., Udry, S., van Kesteren, A., Weber, L., Weilenmann, U.: Setting new standards with HARPS. Messenger 114, 20-24 (2003)

30. Mortier, A., Santos, N.C., Sousa, S.G., Fernand es, J.M., Adibekyan, V.Z., Delgado Mena, E., Montalto, M., Israelian, G.: New and updated stellar parameters for 90 transit hosts. The effect of the surface gravity. A\&A 558, A106 (2013). https://doi.org/10.1051/0004-6361/201322240

31. Mortier, A., Sousa, S.G., Adibekyan, V.Z., Brand ão, I.M., Santos, N.C.: Correcting the spectroscopic surface gravity using transits and asteroseismology. No significant effect on temperatures or 
metallicities with ARES and MOOG in local thermodynamic equilibrium. A\&A 572, A95 (2014). https://doi.org/10.1051/0004-6361/201424537

32. Pepe, F.A., Cristiani, S., Rebolo Lopez, R., Santos, N.C., Amorim, A., Avila, G., Benz, W., Bonifacio, P., Cabral, A., Carvas, P., Cirami, R., Coelho, J., Comari, M., Coretti, I., De Caprio, V., Dekker, H., Delabre, B., Di Marcantonio, P., D’Odorico, V., Fleury, M., García, R., Herreros Linares, J.M., Hughes, I., Iwert, O., Lima, J., Lizon, J.L., Lo Curto, G., Lovis, C., Manescau, A., Martins, C., Mégevand, D., Moitinho, A., Molaro, P., Monteiro, M., Monteiro, M., Pasquini, L., Mordasini, C., Queloz, D., Rasilla, J.L., Rebordão, J.M., Santana Tschudi, S., Santin, P., Sosnowska, D., Spanò, P., Tenegi, F., Udry, S., Vanzella, E., Viel, M., Zapatero Osorio, M.R.: ESPRESSO: the Echelle spectrograph for rocky exoplanets and stable spectroscopic observations. In: SPIE, Society of Photo-Optical Instrumentation Engineers (SPIE) Conference Series, vol. 7735, pp 77350F (2010). https://doi.org/10.1117/12.857122

33. Perryman, M., Hartman, J., Bakos, G.Á.., Lindegren, L.: Astrometric exoplanet detection with Gaia. ApJ 797(1), 14 (2014). https://doi.org/10.1088/0004-637X/797/1/14

34. Pinhas, A., Madhusudhan, N., Gandhi, S., MacDonald, $\mathrm{R} .: \mathrm{H}_{2} \mathrm{O}$ abundances and cloud properties in ten hot giant exoplanets. MNRAS 482(2), 1485-1498 (2019). https://doi.org/10.1093/mnras/sty2544

35. Pollacco, D.L., Skillen, I., Collier Cameron, A., Christian, D.J., Hellier, C., Irwin, J., Lister, T.A., Street, R.A., West, R.G., Anderson, D.R., Clarkson, W.I., Deeg, H., Enoch, B., Evans, A., Fitzsimmons, A., Haswell, C.A., Hodgkin, S., Horne, K., Kane, S.R., Keenan, F.P., Maxted, P.F.L., Norton, A.J., Osborne, J., Parley, N.R., Ryans, R.S.I., Smalley, B., Wheatley, P.J., Wilson, D.M.: The WASP project and the superWASP cameras. PASP 118(848), 1407-1418 (2006). https://doi.org/10.1086/508556

36. Quirrenbach, A., Amado, P.J., Caballero, J.A., Mundt, R., Reiners, A., Ribas, I., Seifert, W., Abril, M., Aceituno, J., Alonso-Floriano, F.J., Anwand -Heerwart, H., Azzaro, M., Bauer, F., Barrado, D., Becerril, S., Bejar, V.J.S., Benitez, D., Berdinas, Z.M., Brinkmöller, M., Cardenas, M.C., Casal, E., Claret, A., Colomé, J., Cortes-Contreras, M., Czesla, S., Doellinger, M., Dreizler, S., Feiz, C., Fernandez, M., Ferro, I.M., Fuhrmeister, B., Galadi, D., Gallardo, I., Gálvez-Ortiz, M.C., Garcia-Piquer, A., Garrido, R., Gesa, L., Gómez Galera, V., González Hernández, J.I., Gonzalez Peinado, R., Grözinger, U., Guàrdia, J., Guenther, E.W., de Guindos, E., Hagen, H.J., Hatzes, A.P., Hauschildt, P.H., Helmling, J., Henning, T., Hermann, D., Hernández Arabi, R., Hernández Castaño, L., Hernández Hernando, F., Herrero, E., Huber, A., Huber, K.F., Huke, P., Jeffers, S.V., de Juan, E., Kaminski, A., Kehr, M., Kim, M., Klein, R., Klüter, J., Kürster, M., Lafarga, M., Lara, L.M., Lamert, A., Laun, W., Launhardt, R., Lemke, U., Lenzen, R., Llamas, M., Lopez del Fresno, M., López-Puertas, M., López-Santiago, J., Lopez Salas, J.F., Magan Madinabeitia, H., Mall, U., Mandel, H., Mancini, L., Marin Molina, J.A., Maroto Fernández, D., Martín, E.L., Martín-Ruiz, S., Marvin, C., Mathar, R.J., Mirabet, E., Montes, D., Morales, J.C., Morales Muñoz, R., Nagel, E., Naranjo, V., Nowak, G., Palle, E., Panduro, J., Passegger, V.M., Pavlov, A., Pedraz, S., Perez, E., Pérez-Medialdea, D., Perger, M., Pluto, M., Ramón, A., Rebolo, R., Redondo, P., Reffert, S., Reinhart, S., Rhode, P., Rix, H.W., Rodler, F., Rodríguez, E., Rodríguez López, C., Rohloff, R.R., Rosich, A., Sanchez Carrasco, M.A., Sanz-Forcada, J., Sarkis, P., Sarmiento, L.F., Schäfer, S., Schiller, J., Schmidt, C., Schmitt, J.H.M.M., Schöfer, P., Schweitzer, A., Shulyak, D., Solano, E., Stahl, O., Storz, C., Tabernero, H.M., Tala, M., Tal-Or, L., Ulbrich, R.G., Veredas, G., Vico Linares, J.I., Vilardell, F., Wagner, K., Winkler, J., Zapatero Osorio, M.R., Zechmeister, M., Ammler-von Eiff, M., Anglada-Escudé, G., del Burgo, C., Garcia-Vargas, M.L., Klutsch, A., Lizon, J.L., Lopez-Morales, M., Ofir, A., Pérez-Calpena, A., Perryman, M.A.C., Sánchez-Blanco, E., Strachan, J.B.P., Stürmer, J., Suárez, J.C., Trifonov, T., Tulloch, S.M., Xu, W.: CARMENES: an overview six months after first light. In: Ground-based and Airborne Instrumentation for Astronomy VI, Society of Photo-Optical Instrumentation Engineers (SPIE) Conference Series, vol. 9908, pp 990812 (2016). https://doi.org/10.1117/12.2231880

37. Rauer, H., Aerts, C., Cabrera, J., Team, P.LATO.: The PLATO mission. Astronomische Nachr. 337(89), 961 (2016). https://doi.org/10.1002/asna.201612408

38. Ricker, G.R., Winn, J.N., Vanderspek, R., Latham, D.W., Bakos, G.Á.., Bean, J.L., Berta-Thompson, Z.K., Brown, T.M., Buchhave, L., Butler, N.R., Butler, R.P., Chaplin, W.J., Charbonneau, D., Christensen-Dalsgaard, J., Clampin, M., Deming, D., Doty, J., De Lee, N., Dressing, C., Dunham, E.W., Endl, M., Fressin, F., Ge, J., Henning, T., Holman, M.J., Howard, A.W., Ida, S., Jenkins, J., Jernigan, G., Johnson, J.A., Kaltenegger, L., Kawai, N., Kjeldsen, H., Laughlin, G., Levine, A.M., Lin, D., Lissauer, J.J., MacQueen, P., Marcy, G., McCullough, P.R., Morton, T.D., Narita, N., Paegert, M., Palle, E., Pepe, F., Pepper, J., Quirrenbach, A., Rinehart, S.A., Sasselov, D., Sato, B., Seager, S., 
Sozzetti, A., Stassun, K.G., Sullivan, P., Szentgyorgyi, A., Torres, G., Udry, S., Villasenor, J.: Transiting exoplanet survey satellite (TESS). In: SPIE, Society of Photo-Optical Instrumentation Engineers (SPIE) Conference Series, vol. 9143, pp 914320 (2014). https://doi.org/10.1117/12.2063489

39. Robertson, P., Endl, M., Henry, G.W., Cochran, W.D., MacQueen, P.J., Williamson, M.H.: Stellar activity and its implications for exoplanet detection on GJ 176. ApJ 801(2), 79 (2015). https://doi.org/10.1088/0004-637X/801/2/79

40. Ryabchikova, T., Piskunov, N., Kurucz, R.L., Stempels, H.C., Heiter, U., Pakhomov, Y., Barklem, P.S.: A major upgrade of the VALD database. Phys. Scr. 90(5), 054,005 (2015). https://doi.org/10.1088/ 0031-8949/90/5/054005

41. Santos, N.C., Israelian, G., Mayor, M.: Spectroscopic [Fe/H] for 98 extra-solar planet-host stars. Exploring the probability of planet formation. A\&A 415, 1153-1166 (2004). https://doi.org/10.1051/ 0004-6361:20034469

42. Santos, N.C., Sousa, S.G., Mortier, A., Neves, V., Adibekyan, V., Tsantaki, M., Delgado Mena, E., Bonfils, X., Israelian, G., Mayor, M., Udry, S.: SWEET-Cat: A catalogue of parameters for Stars With ExoplanETs. I. New atmospheric parameters and masses for 48 stars with planets. A\&A 556, A150 (2013). https://doi.org/10.1051/0004-6361/201321286

43. Schlaufman, K.C.: A continuum of planet formation between 1 and 4 Earth Radii. ApJ 799(2), L26 (2015). https://doi.org/10.1088/2041-8205/799/2/L26

44. Sing, D.K., Fortney, J.J., Nikolov, N., Wakeford, H.R., Kataria, T., Evans, T.M., Aigrain, S., Ballester, G.E., Burrows, A.S., Deming, D., Désert, J.M., Gibson, N.P., Henry, G.W., Huitson, C.M., Knutson, H.A., Lecavelier Des Etangs, A., Pont, F., Showman, A.P., Vidal-Madjar, A., Williamson, M.H.: Wilson, P.A.: A continuum from clear to cloudy hot-Jupiter exoplanets without primordial water depletion. Nature 529(7584), 59-62 (2016). https://doi.org/10.1038/nature16068

45. Smiljanic, R., Korn, A.J., Bergemann, M., Frasca, A., Magrini, L., Masseron, T., Pancino, E., Ruchti, G., San Roman, I., Sbordone, L., Sousa, S.G., Tabernero, H., Tautvaiv̌ienė, G., Valentini, M., Weber, M., Worley, C.C., Adibekyan, V.Z., Allende Prieto, C., Barisevičius, G., Biazzo, K., BlancoCuaresma, S., Bonifacio, P., Bragaglia, A., Caffau, E., Cantat-Gaudin, T., Chorniy, Y., de Laverny, P., Delgado-Mena, E., Donati, P., Duffau, S., Franciosini, E., Friel, E., Geisler, D., GonzáTautvailez Hernández, J.I., Gruyters, P., Guiglion, G., Hansen, C.J., Heiter, U., Hill, V., Jacobson, H.R., Jofre, P., Jönsson, H., Lanzafame, A.C., Lardo, C., Ludwig, H.G., Maiorca, E., Mikolaitis, Š., Montes, D., Morel, T., Mucciarelli, A., Muñoz, C., Nordland er, T., Pasquini, L., Puzeras, E., Recio-Blanco, A., Ryde, N., Sacco, G., Santos, N.C., Serenelli, A.M., Sordo, R., Soubiran, C., Spina, L., Steffen, M., Vallenari, A., Van Eck, S., Villanova, S., Gilmore, G., Randich, S., Asplund, M., Binney, J., Drew, J., Feltzing, S., Ferguson, A., Jeffries, R., Micela, G., Negueruela, I., Prusti, T., Rix, H.W., Alfaro, E., Babusiaux, C., Bensby, T., Blomme, R., Flaccomio, E., Franciois, P., Irwin, M., Koposov, S., Walton, N., Bayo, A., Carraro, G., Costado, M.T., Damiani, F., Edvardsson, B., Hourihane, A., Jackson, R., Lewis, J., Lind, K., Marconi, G., Martayan, C., Monaco, L., Morbidelli, L., Prisinzano, L., Zaggia, S.: The Gaia-ESO Survey: The analysis of high-resolution UVES spectra of FGK-type stars. A\&A 570, A122 (2014). https://doi.org/10.1051/0004-6361/201423937

46. Sneden, C.: The nitrogen abundance of the very metal-poor star HD 122563. AJ 184, 839 (1973). https://doi.org/10.1086/152374

47. Sousa, S.G., Adibekyan, V., Delgado-Mena, E., Santos, N.C., Andreasen, D.T., Ferreira, A.C.S., Tsantaki, M., Barros, S.C.C., Demangeon, O., Israelian, G., Faria, J.P., Figueira, P., Mortier, A., Brandão, I., Montalto, M., Rojas-Ayala, B., Santerne, A.: SWEET-Cat updated. New homogenous spectroscopic parameters. A\&A 620, A58 (2018). https://doi.org/10.1051/0004-6361/201833350

48. Sousa, S.G., Santos, N.C., Israelian, G., Mayor, M., Monteiro, M.J.P.G.: A new code for automatic determination of equivalent widths: Automatic routine for line equivalent widths in stellar spectra (ARES). A\&A 469(2), 783-791 (2007). https://doi.org/10.1051/0004-6361:20077288

49. Sousa, S.G., Santos, N.C., Mayor, M., Udry, S., Casagrande, L., Israelian, G., Pepe, F., Queloz, D., Monteiro, M.J.P.F.G.: Spectroscopic parameters for 451 stars in the HARPS GTO planet search program. Stellar $[\mathrm{Fe} / \mathrm{H}]$ and the frequency of exo-Neptunes. A\&A 487(1), 373-381 (2008). https://doi.org/10.1051/0004-6361:200809698

50. Sousa, S.G., Santos, N.C., Mortier, A., Tsantaki, M., Adibekyan, V., Delgado Mena, E., Israelian, G., Rojas-Ayala, B., Neves, V.: Homogeneous spectroscopic parameters for bright planet host stars from the northern hemisphere. The impact on stellar and planetary mass. AAp 576, A94 (2015). https://doi.org/10.1051/0004-6361/201425227

51. Sozzetti, A.: The Gaia astrometric survey. Highlights Astron. 15, 716-717 (2010). https://doi.org/10. $1017 /$ S1743921310011142 
52. Sozzetti, A., Torres, G., Charbonneau, D., Latham, D.W., Holman, M.J., Winn, J.N., Laird, J.B., O'Donovan, F.T.: Improving stellar and planetary parameters of transiting planet systems: The Case of TrES-2. ApJ 664(2), 1190-1198 (2007). https://doi.org/10.1086/519214

53. Stetson, P.B., Pancino, E.: DAOSPEC: An automatic code for measuring equivalent widths in highresolution stellar spectra. PASP 120(874), 1332 (2008). https://doi.org/10.1086/596126

54. Stevenson, K.B., Désert, J.M., Line, M.R., Bean, J.L., Fortney, J.J., Showman, A.P., Kataria, T., Kreidberg, L., McCullough, P.R., Henry, G.W., Charbonneau, D., Burrows, A., Seager, S., Madhusudhan, N., Williamson, M.H., Homeier, D.: Thermal structure of an exoplanet atmosphere from phase-resolved emission spectroscopy. Science 346(6211), 838-841 (2014). https://doi.org/10.1126/science. 1256758

55. Tinetti, G., Drossart, P., Eccleston, P., Hartogh, P., Heske, A., Leconte, J., Micela, G., Ollivier, M., Pilbratt, G., Puig, L., Turrini, D., Vandenbussche, B., Wolkenberg, P., Beaulieu, J.P., Buchave, L.A., Ferus, M., Griffin, M., Guedel, M., Justtanont, K., Lagage, P.O., Machado, P., Malaguti, G., Min, M., Nórgaard-Nielsen, H.U., Rataj, M., Ray, T., Ribas, I., Swain, M., Szabo, R., Werner, S., Barstow, J., Burleigh, M., Cho, J., du Foresto, V.C., Coustenis, A., Decin, L., Encrenaz, T., Galand, M., Gillon, M., Helled, R., Morales, J.C., Muñoz, A.G., Moneti, A., Pagano, I., Pascale, E., Piccioni, G., Pinfield, D., Sarkar, S., Selsis, F., Tennyson, J., Triaud, A., Venot, O., Waldmann, I., Waltham, D., Wright, G., Amiaux, J., Auguères, J.L., Berthé, M., Bezawada, N., Bishop, G., Bowles, N., Coffey, D., Colomé, J., Crook, M., Crouzet, P.E., Da Peppo, V., Sanz, I.E., Focardi, M., Frericks, M., Hunt, T., Kohley, R., Middleton, K., Morgante, G., Ottensamer, R., Pace, E., Pearson, C., Stamper, R., Symonds, K., Rengel, M., Renotte, E., Ade, P., Affer, L., Alard, C., Allard, N., Altieri, F., André, Y., Arena, C., Argyriou, I., Aylward, A., Baccani, C., Bakos, G., Banaszkiewicz, M., Barlow, M., Batista, V., Bellucci, G., Benatti, S., Bernardi, P., Bézard, B., Blecka, M., Bolmont, E., Bonfond, B., Bonito, R., Bonomo, A.S., Brucato, J.R., Brun, A.S., Bryson, I., Bujwan, W., Casewell, S., Charnay, B., Pestellini, C.C., Chen, G., Ciaravella, A., Claudi, R., Clédassou, R., Damasso, M., Damiano, M., Danielski, C., Deroo, P., Di Giorgio, A.M., Dominik, C., Doublier, V., Doyle, S., Doyon, R., Drummond, B., Duong, B., Eales, S., Edwards, B., Farina, M., Flaccomio, E., Fletcher, L., Forget, F., Fossey, S., Fränz, M., Fujii, Y., García-Piquer, Á., Gear, W., Geoffray, H., Gérard, J.C., Gesa, L., Gomez, H., Graczyk, R., Griffith, C., Grodent, D., Guarcello, M.G., Gustin, J., Hamano, K., Hargrave, P., Hello, Y., Heng, K., Herrero, E., Hornstrup, A., Hubert, B., Ida, S., Ikoma, M., Iro, N., Irwin, P., Jarchow, C., Jaubert, J., Jones, H., Julien, Q., Kameda, S., Kerschbaum, F., Kervella, P., Koskinen, T., Krijger, M., Krupp, N., Lafarga, M., Landini, F., Lellouch, E., Leto, G., Luntzer, A., Rank-Lüftinger, T., Maggio, A., Maldonado, J., Maillard, J.P., Mall, U., Marquette, J.B., Mathis, S., Maxted, P., Matsuo, T., Medvedev, A., Miguel, Y., Minier, V., Morello, G., Mura, A., Narita, N., Nascimbeni, V., Nguyen Tong, N., Noce, V., Oliva, F., Palle, E., Palmer, P., Pancrazzi, M., Papageorgiou, A., Parmentier, V., Perger, M., Petralia, A., Pezzuto, S., Pierrehumbert, R., Pillitteri, I., Piotto, G., Pisano, G., Prisinzano, L., Radioti, A., Réess, J.M., Rezac, L., Rocchetto, M., Rosich, A., Sanna, N., Santerne, A., Savini, G., Scandariato, G., Sicardy, B., Sierra, C., Sindoni, G., Skup, K., Snellen, I., Sobiecki, M., Soret, L., Sozzetti, A., Stiepen, A., Strugarek, A., Taylor, J., Taylor, W., Terenzi, L., Tessenyi, M., Tsiaras, A., Tucker, C., Valencia, D., Vasisht, G., Vazan, A., Vilardell, F., Vinatier, S., Viti, S., Waters, R., Wawer, P., Wawrzaszek, A., Whitworth, A., Yung, Y.L., Yurchenko, S.N., Osorio, M.R.Z., Zellem, R., Zingales, T., Zwart, F.: A chemical survey of exoplanets with ARIEL. Exp. Astron. 46(1), 135-209 (2018). https://doi.org/10.1007/s10686-018-9598-X

56. Torres, G., Andersen, J., Giménez, A.: Accurate masses and radii of normal stars: modern results and applications. A\&A Rev. 18(1-2), 67-126 (2010). https://doi.org/10.1007/s00159-009-0025-1

57. Torres, G., Fischer, D.A., Sozzetti, A., Buchhave, L.A., Winn, J.N., Holman, M.J., Carter, J.A.: Improved spectroscopic parameters for transiting planet hosts. ApJ 757(2), 161 (2012). https://doi.org/10.1088/0004-637X/757/2/161

58. Tsantaki, M., Andreasen, D., Teixeira, G.: FASMA 2.0: A Python package for stellar parameters and chemical abundances. J. Open Source Softw. 5(50), 2048 (2020). https://doi.org/10.21105/joss.02048

59. Tsantaki, M., Andreasen, D.T., Teixeira, G.D.C., Sousa, S.G., Santos, N.C., Delgado-Mena, E., Bruzual, G.: Atmospheric stellar parameters for large surveys using FASMA, a new spectral synthesis package. MNRAS 473(4), 5066-5097 (2018). https://doi.org/10.1093/mnras/stx2564

60. Tsantaki, M., Sousa, S.G., Adibekyan, V.Z., Santos, N.C., Mortier, A., Israelian, G.: Deriving precise parameters for cool solar-type stars. Optimizing the iron line list. A\&A 555, A150 (2013). https://doi.org/10.1051/0004-6361/201321103 
61. Tsantaki, M., Sousa, S.G., Santos, N.C., Montalto, M., Delgado-Mena, E., Mortier, A., Adibekyan, V., Israelian, G.: Spectroscopic parameters for solar-type stars with moderate-to-high rotation. New parameters for ten planet hosts. A\&A 570, A80 (2014). https://doi.org/10.1051/0004-6361/201424257

62. Tsiaras, A., Waldmann, I.P., Zingales, T., Rocchetto, M., Morello, G., Damiano, M., Karpouzas, K., Tinetti, G., McKemmish, L.K., Tennyson, J., Yurchenko, S.N.: A population study of gaseous exoplanets. AJ 155(4), 156 (2018). https://doi.org/10.3847/1538-3881/aaaf75

63. Turrini, D., Miguel, Y., Zingales, T., Piccialli, A., Helled, R., Vazan, A., Oliva, F., Sindoni, G., Panić, O., Leconte, J., Min, M., Pirani, S., Selsis, F., Coudé du Foresto, V., Mura, A., Wolkenberg, P.: The contribution of the ARIEL space mission to the study of planetary formation. Exp. Astron. 46(1), 45-65 (2018). https://doi.org/10.1007/s10686-017-9570-1

64. Valenti, J.A., Fischer, D.A.: Spectroscopic Properties of Cool Stars (SPOCS). I. 1040 F, G, and K Dwarfs from Keck. Lick, and AAT Planet Search Programs. ApJS 159(1), 141-166 (2005). https://doi.org/10.1086/430500

65. Wagner, F.W., Sohl, F., Hussmann, H., Grott, M., Rauer, H.: Interior structure models of solid exoplanets using material laws in the infinite pressure limit. Icarus 214(2), 366-376 (2011). https://doi.org/10.1016/j.icarus.2011.05.027

66. Wheatley, P.J., Pollacco, D.L., Queloz, D., Rauer, H., Watson, C.A., West, R.G., Chazelas, B., Louden, T.M., Walker, S., Bannister, N., Bento, J., Burleigh, M., Cabrera, J., Eigmüller, P., Erikson, A., Genolet, L., Goad, M., Grange, A., Jordán, A., Lawrie, K., McCormac, J., Neveu, M.: The next generation transit survey (NGTS). In: European Physical Journal Web of Conferences, European Physical Journal Web of Conferences, vol. 47, pp 13002 (2013). https://doi.org/10.1051/epjconf/20134713002

67. Wilson, R.F., Teske, J., Majewski, S.R., Cunha, K., Smith, V., Souto, D., Bender, C., Mahadevan, S., Troup, N., Allende Prieto, C., Stassun, K.G., Skrutskie, M.F., Almeida, A., García-Hernández, D.A., Zamora, O., Brinkmann, J.: Elemental abundances of kepler objects of interest in APOGEE. I. Two distinct orbital period regimes inferred from host star iron abundances. AJ 155(2), 68 (2018). https://doi.org/10.3847/1538-3881/aa9f27

68. Zhang, Z., Zhou, Y., Rackham, B.V., Apai, D.: The near-infrared transmission spectra of TRAPPIST1 Planets b, c, d, e, f, and g and stellar contamination in multi-epoch transit spectra. AJ 156(4), 178 (2018). https://doi.org/10.3847/1538-3881/aade4f

69. Zingales, T., Tinetti, G., Pillitteri, I., Leconte, J., Micela, G., Sarkar, S.: The ARIEL mission reference sample. Exp. Astron. 46(1), 67-100 (2018). https://doi.org/10.1007/s10686-018-9572-7

Publisher's note Springer Nature remains neutral with regard to jurisdictional claims in published maps and institutional affiliations.

\section{Affiliations}

\section{Anna Brucalassi ${ }^{1}$ (1) - Maria Tsantaki ${ }^{1}$. Laura Magrini ${ }^{1}$. Sergio Sousa ${ }^{2}$. Camilla Danielski ${ }^{3}$. Katia Biazzo ${ }^{4}$. Giada Casali ${ }^{1,5}$. Mathieu Van der Swaelmen ${ }^{1}$. Monica Rainer ${ }^{1}$. Vardan Adibekyan ${ }^{2}$. Elisa Delgado-Mena ${ }^{2} \cdot$ Nicoletta Sanna ${ }^{1}$}

1 INAF-Osservatorio Astronomico di Arcetri, Largo Enrico Fermi 5, 50125 Firenze, Italy

2 Instituto de Astrofísica e Ciências do Espaço, Universidade do Porto, CAUP, Rua das Estrelas, 4150-762 Porto, Portugal

3 UCL Centre for Space Exochemistry Data Atlas Building, Fermi Avenue, Harwell Campus 1 Didcot 1, OX11 0QR, London, UK

4 INAF-Osservatorio Astronomico di Roma, Via di Frascati 33, 00044 Monte Porzio Catone, Italy

5 Dipartimento di Fisica e Astronomia, Università degli Studi di Firenze, via G. Sansone 1, 50019 Sesto Fiorentino (Firenze), Italy 